\title{
OPEN Triazine-based porous organic polymers for reversible capture of iodine and utilization in antibacterial application
}

\begin{abstract}
Anandhu Mohan, Mohammad H. Al-Sayah, Abdelrahman Ahmed \& Oussama M. El-Kadri ${ }^{\bowtie}$
The capture and safe storage of radioactive iodine $\left({ }^{129} \mathrm{I}\right.$ or $\left.{ }^{131} \mathrm{I}\right)$ are of a compelling significance in the generation of nuclear energy and waste storage. Because of their physiochemical properties, Porous Organic Polymers (POPs) are considered to be one of the most sought classes of materials for iodine capture and storage. Herein, we report on the preparation and characterization of two triazine-based, nitrogen-rich, porous organic polymers, NRPOP-1 $\left(\mathrm{SA}_{\mathrm{BET}}=519 \mathrm{~m}^{2} \mathrm{~g}^{-1}\right)$ and NRPOP-2 $(\mathrm{SA} \mathrm{BET}=456$ $\left.\mathrm{m}^{2} \mathrm{~g}^{-1}\right)$, by reacting 1,3,5-triazine-2,4,6-triamine or 1,4-bis-(2,4-diamino-1,3,5-triazine)-benzene with thieno[2,3-b]thiophene-2,5-dicarboxaldehyde, respectively, and their use in the capture of volatile iodine. NRPOP-1 and NRPOP-2 showed a high adsorption capacity of iodine vapor with an uptake of up to $317 \mathrm{wt} \%$ at $80^{\circ} \mathrm{C}$ and 1 bar and adequate recyclability. The NRPOPs were also capable of removing up to $87 \%$ of iodine from $300 \mathrm{mg} \mathrm{L}^{-1}$ iodine-cyclohexane solution. Furthermore, the iodineloaded polymers, $\mathrm{I}_{2}$ @NRPOP-1 and $\mathrm{I}_{2}$ @NRPOP-2, displayed good antibacterial activity against Micrococcus luteus (ML), Escherichia coli (EC), and Pseudomonas aeruginosa (PSA). The synergic functionality of these novel polymers makes them promising materials to the environment and public health.
\end{abstract}

To reduce the concentration of carbon dioxide $\left(\mathrm{CO}_{2}\right)$ in the atmosphere, which is produced mainly by fossil fuel power plants, and meet the world energy demands, many countries have optioned for nuclear energy as an alternative to fossil fuels given its high energy density, minimal carbon footprints, and low working cost ${ }^{1-3}$. Despite such advantages, exhaust fumes from nuclear power plants contain a significant amount of radioactive species that include ${ }^{129} \mathrm{I}$ and ${ }^{131} \mathrm{I}$, leading to major environmental and biological implications $\mathrm{s}^{3,4}$. It has been projected by the IAEA that nuclear electricity capacity will almost double by 2050 , and therefore, necessitates the need to develop highly efficient materials for the capture and storage of radioactive species ${ }^{5-7}$. Inorganic composite materials like silver-based zeolite have been widely used in radioactive iodine capture ${ }^{6,7}$. However, such materials suffer from low iodine uptake capacity due to their limited surface area and high cost. Therefore, the development of novel adsorbent materials for iodine capture is of significant importance.

Iodine is widely used as a powerful and effective disinfectant owing to its ability to destroy a wide range of living disease-causing bacteria ${ }^{8,9}$. Nevertheless, it is unsafe when directly applied to wounds owning to its instability and high volatility ${ }^{10-12}$. Recently, the use of polyvinylpyrrolidone (PVP) as an adsorbent for iodine has been reported. Such polymers can stabilize iodine through complexation and readily release it and hence, exterminating pathogenic microorganisms like bacteria, virus, and fungi ${ }^{13,14}$. The major disadvantages of these types of polymers are the low performance in water medium because of their high solubility and the somewhat complicated synthetic routes that limit their scale-up production ${ }^{15,16}$.

Porous organic polymers (POPs) have emerged as potential adsorbents for many pollutants including $\mathrm{CO}_{2}$, heavy metals, organic vapors, dyes, etc ${ }^{17-22}$. Constructed from light atoms $(\mathrm{H}, \mathrm{C}, \mathrm{N}, \mathrm{O})$, POPs often show excellent thermal and chemical stabilities, high porosity, and tuneable chemical functionality, and thus can be easily tailored toward targeted applications ${ }^{23}$. POPs have been reported to be effective absorbents for revisable iodine sequestration with varying uptake capacity, release, and recyclability; however, the reutilization of the encapsulated iodine for potential applications has not been explored. Our group is interested in the development of triazine-based, aminal-linked porous organic polymers because they offer an optimized environment for the capture and storage of guest molecules through the introduction of chemical functional units via cost effective, 
metal-free condensation reactions. Previously, we reported on the synthesis of a number of nitrogen-rich aminallinked POPs (NRAPOPs) that displayed excellent iodine and $\mathrm{CO}_{2}$ capturing capacities ${ }^{24,25}$. We attributed the high $\mathrm{I}_{2}$ and $\mathrm{CO}_{2}$ capture to the simultaneous effect of the high content of heteroatoms, porosity, and rich- $\pi$-electron conjugated structures, which contribute to the enhancement of the adsorbate-adsorbent interactions ${ }^{26,27}$. In this study, we extended the NRAPOPs library by preparing two cost-effective-novel porous polymers, NRPOP-1 and NRPOP-2, through metal free-Schiff base polycondensation reaction of 1,3,5-triazine-2,4,6-triamine (melamine) or 1,4-bis-(2,4-diamino-1,3,5-triazine)-benzene with thieno[2,3-b]thiophene-2,5-dicarboxaldehyde. The obtained porous polymers exhibit high iodine vapor capturing capacity of up to $317 \mathrm{wt} \%$ and have over $87 \%$ iodine removal efficiency from solution. The effects of heteroatoms and phenyl rings on the chemical and structural properties of the polymers and their contribution to iodine adsorption are discussed. Aside from their excellent iodine adsorption, the iodine-loaded NRPOPs ( $\left.\mathrm{I}_{2} @ N R P O P s\right)$ showed good antibacterial effectiveness against a wide range of bacteria that included Micrococcus luteus (ML), Escherichia coli (EC), and Pseudomonas aeruginosa (PSA) through their release of iodine. To the best of our knowledge, NRPOP-1 and NRPOP-2, present the first examples of porous organic polymers to utilize the encapsulated iodine in the antibacterial application. Our study herein suggests that other classes of porous materials, such as metal organic frameworks (MOFs), would also be reliable adsorbents for the capture and reutilization of pollutants in the fields of public health and chemical industry.

\section{Experimental section}

Material. All solvents and starting materials were bought from commercial suppliers (Sigma-Aldrich, Acros Organics, and Frontier Scientifics) and used without further purification. 1,4-bis-(2,4-diamino-1,3,5-triazine) benzene was prepared according to a previously reported procedure ${ }^{28}$.

Synthesis of NRPOP-1. NRPOP-1 was synthesized by reacting $0.350 \mathrm{~g}(1.78 \mathrm{mmol})$ of thieno[2,3-b]thiophene-2,5-dicarboxaldehyde with $0.150 \mathrm{~g}(1.19 \mathrm{mmol})$ of 1,3,5-triazine-2,4,6-triamine (melamine) in $14.8 \mathrm{~mL}$ of anhydrous dimethyl sulfoxide (DMSO) at $180^{\circ} \mathrm{C}$ for $72 \mathrm{~h}$ under argon atmosphere. The mixture was filtered, and the obtained solid was washed with tetrahydrofuran (THF), methanol, and acetone and dried under vacuum overnight to give a yellow-colored powder in $76 \%$ yield.

Synthesis of NRPOP-2. In a similar fashion to the preparation of NRPOP-1, NRPOP-2 was synthesized by reacting $0.350 \mathrm{~g}(1.78 \mathrm{mmol})$ of thieno[2,3-b]thiophene-2,5-dicarboxaldehyde with $0.264 \mathrm{~g}(0.891 \mathrm{mmol})$ of 1,4-bis-(2,4-diamino-1,3,5-triazine)-benzene in $13.5 \mathrm{~mL}$ of anhydrous DMSO to give a light yellow-colored powder in $73 \%$ yield.

lodine adsorption and release studies. The gravimetric measurements based on iodine adsorption experiments were carried out by the following procedure: $40 \mathrm{mg}$ of each polymer (NRPOP-1 and NRPOP-2) was taken in separate $10 \mathrm{~mL}$ glass vials. After noting the initial weight, the vials were placed in a glass jar container having an excess amount of solid iodine. The glass jar was then sealed and heated at $353 \mathrm{~K}$ and 1.0 bar in an oven. After the iodine adsorption, the system was allowed to cool down to room temperature and weighed afterward. The iodine adsorption capacities for the NRPOPs were calculated by the following equation:

$$
\frac{\mathrm{m}_{2}-\mathrm{m}_{1}}{\mathrm{~m}_{1}} * 100 \mathrm{wt} \%
$$

where $\mathrm{m}_{1}$ and $\mathrm{m}_{2}$ are masses of polymer before and after adsorption. The adsorption isotherm and capacity of the dissolved iodine were evaluated by placing $3 \mathrm{mg}$ of fresh NRAPOPs in $0.3 \mathrm{~g} \mathrm{~mL}^{-1}$ iodine-cyclohexane solution. The progress of the iodine adsorption was checked by UV-Vis spectrometer. The iodine release experiments were carried out using ethanol as the solvent. For that, $50 \mathrm{~mL}$ of ethanol was added to a conical flask containing the $5 \mathrm{mg}$ of the iodine-loaded NRPOPs, and the extent of iodine released was monitored by UV-Vis spectroscopy at specific time intervals.

Antibacterial activity of the iodine captured polymers. The antibacterial activity of the $\mathrm{I}_{2} @ \mathrm{NRPOPs}$ was assessed against three bacteria species: Escherichia coli (EC, MicroKwik Culture \# 155065A), Pseudomonas aeruginosa (PSA, MicroKwik Culture \# 155250A), and Micrococcus luteus (ML, MicroKwik Culture \# 155155A) using agar diffusion test. The bacterial inoculum was uniformly spread using a sterile cotton swab on a sterile Petri dish agar. Then, a sample $(1 \mathrm{mg})$ of the iodine-loaded polymers was placed in the center of the dish and it was covered by a sterile wet ( 15 microL of water) paper disk ( $5 \mathrm{~mm}$ in diameter). For the control experiments, a sample of the unloaded polymer was used. After incubation for $24 \mathrm{~h}$ at $37^{\circ} \mathrm{C}$ for EC and PSA, and at $25^{\circ} \mathrm{C}$ for $\mathrm{ML}$, the diameter of the growth inhibition zone was measured to determine the efficiency of the NRPOPs. Tests were performed in triplicates.

Instrumentation and methods. Surface area and pore size distribution of the synthesized material were measured by using nitrogen adsorption and desorption isotherm (Quantochrome analyzer at $77 \mathrm{~K}$ ), which were calculated by using Braunauer-Emmett-Teller (BET) and the non-local density functional theory (NLDFT) methods, respectively. Fourier transform infrared spectra were recorded by Perkin-Elmer FT-IR spectrometer. Thermogravimetric analysis (TGA, Perkin-Elmer thermogravimetric analyzer) was carried out to check the thermal stability of the material at a heating rate of $10{ }^{\circ} \mathrm{C} \mathrm{min}^{-1}$ in a temperature range of $30-800{ }^{\circ} \mathrm{C}$ under air atmosphere. Powder X-ray diffraction (PXRD) patterns were measured in $2 \theta$ range of $5-90^{\circ}$ at a scanning speed 


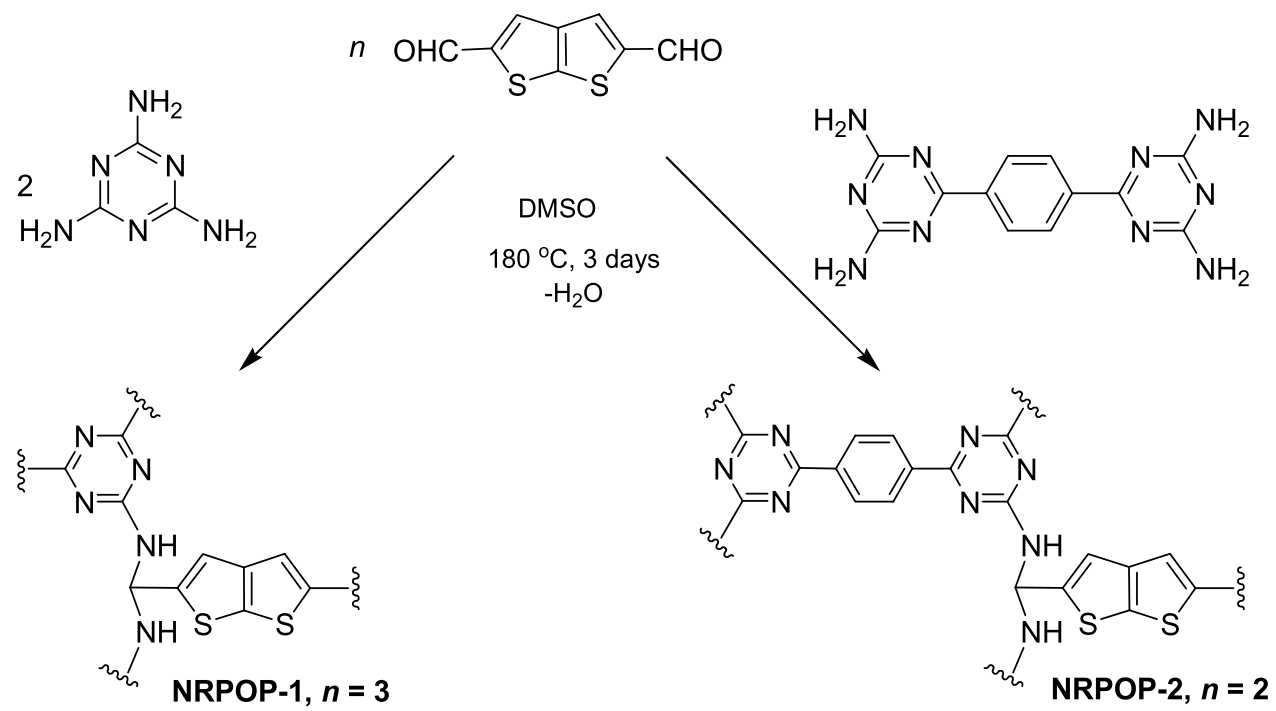

Scheme 1.. Schematic illustration of the synthesis of NRPOP-1 and NRPOP-2.
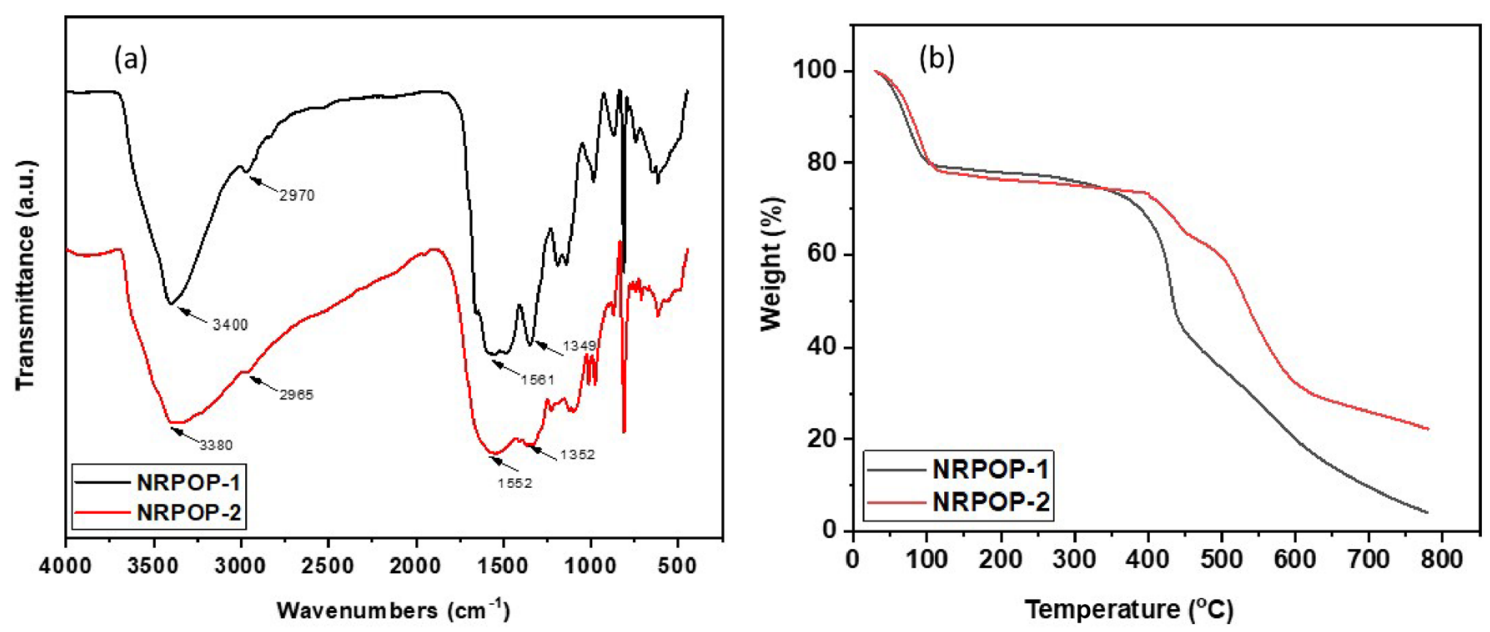

Figure 1. FTIR spectra (a) and TGA (b) of NRPOP-1 and NRPOP-2.

of $5^{\circ} \mathrm{min}^{-1}$ in room temperature by using a Panalytical X'pert pro multipurpose diffractometer having a $\mathrm{Cu}$ $\mathrm{Ka}$ radiation. SEM images were recorded with high resolution scanning electron microscope (TESCAN-LMU SEM). Iodine solution concentrations were measured using a UV-Vis spectrometer (Shimadzu UV-1800 spectrometer) coupled with a quartz cuvette.

\section{Results and discussion}

Synthesis and characterization. The triazine-based nitrogen-rich porous organic polymers (NRPOPs) were synthesized by Schiff-base reaction of 1,3,5-triazine-2,4,6-triamine (melamine) or 1,4-bis-(2,4-diamino1,3,5-triazine)-benzene with thieno[2,3-b]thiophene-2,5-dicarboxaldehyde in DMSO at $180{ }^{\circ} \mathrm{C}$ for $72 \mathrm{~h}$ under argon atmosphere without the need of adding catalysts as depicted in Scheme 1. The obtained dark yellow (NRPOP-1) and light yellow (NRPOP-2) solids were characterized by spectroscopic and analytical techniques. The polymers are insoluble in common solvents, such as DMSO, dichloromethane (DCM), dimethylformamide (DMF), THF), chloroform $\left(\mathrm{CHCl}_{3}\right)$, and ethanol (EtOH), suggesting a high degree of cross-linking, which facilitates their ease of isolation.

The successful formation of the NRPOPs was confirmed by FTIR spectroscopy (Fig. 1a). The aminal linkage formation between the triazine units and the aldehyde monomer is indicated by the appearance of stretching vibration bands at $3400 / 3380$ and $2970 / 2965 \mathrm{~cm}^{-1}$ corresponding to secondary amine $(\mathrm{N}-\mathrm{H})$ and methylene $(\mathrm{C}-\mathrm{H})$ stretching. The inclusion of the triazine building block is confirmed by the presence of characteristic peaks at $1561 / 1552$ and $1349 / 1352 \mathrm{~cm}^{-128,29}$. In addition, the disappearance of the primary amine's $\mathrm{NH}_{2}$ (stretching/deformation) peaks and the aldehyde's stretching band of its $\mathrm{C}=\mathrm{O}$ reveals the consumption of these groups which add another evidence for the formation of the NRPOPs (Fig. S1) ${ }^{30,31}$. The thermal stability of the as-prepared polymers was investigated by thermogravimetric analysis (TGA). Both NRPOPs show excellent 

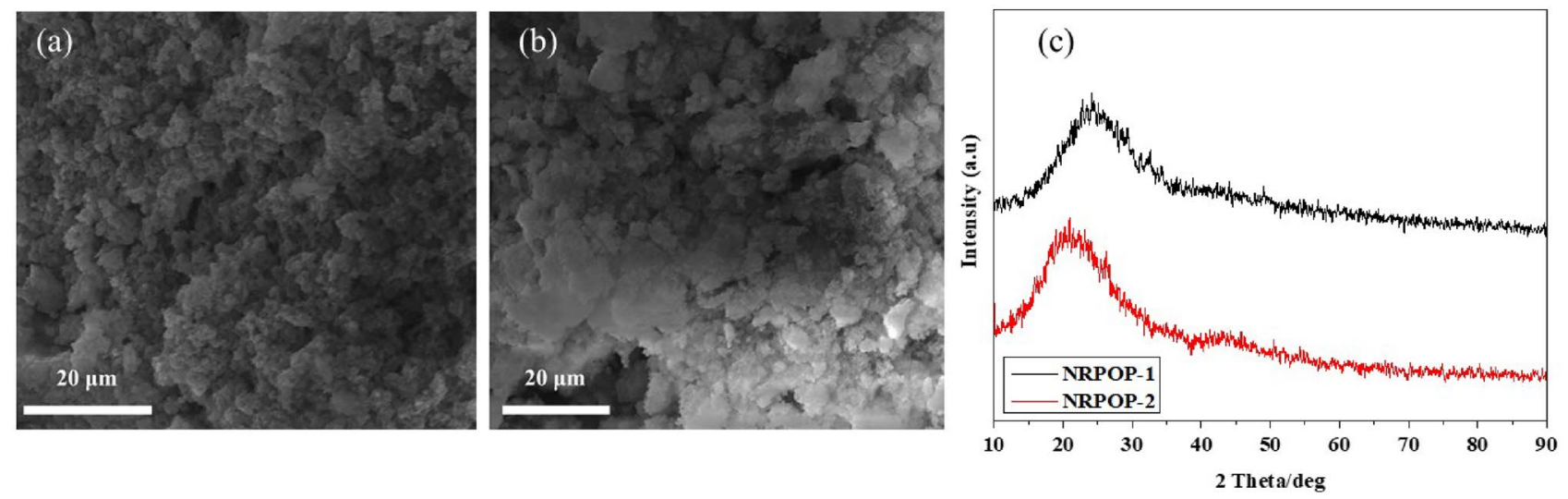

Figure 2. SEM images of NRPOP-1 (a) and NRPOP-2 (b) and their respective PXRD patterns (c).
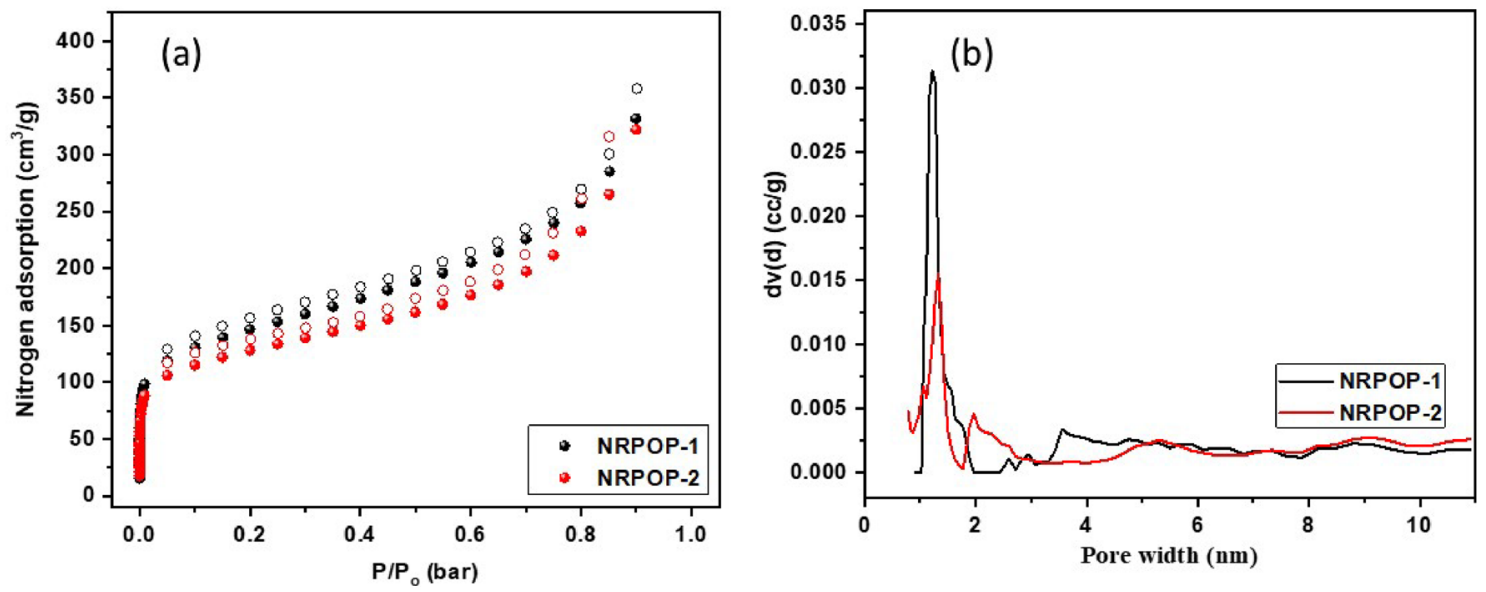

Figure 3. $\mathrm{N}_{2}$ adsorption-desorption isotherms (a) and pore size distribution curves (b) of NRPOP-1 and NRPOP-2.

\begin{tabular}{|l|l|l|l|}
\hline Sample & $\mathbf{S}_{\text {BET }}\left(\mathbf{m}^{\mathbf{2}} \mathbf{g}^{-\mathbf{1}}\right)^{\mathbf{a}}$ & Pore volume $\left(\mathbf{c m}^{\mathbf{3}} \mathbf{g}^{-\mathbf{1}}\right)^{\mathbf{b}}$ & Pore size $(\mathbf{n m})^{\mathbf{c}}$ \\
\hline NRPOP-1 & 519 & 0.703 & 1.2 \\
\hline NRPOP-2 & 456 & 0.675 & 1.3 \\
\hline
\end{tabular}

Table 1. Textural properties of NRPOP-1 and NRPOP-2. ${ }^{a}$ BET surface area. ${ }^{\mathrm{b}}$ Total pore volume at $P / P_{\mathrm{o}}=0.95$. ${ }^{c}$ Dominant pore size distribution calculated by NLDFT.

thermal stability up to about $350^{\circ} \mathrm{C}$ (Fig. 1b) because of the strong covalent interactions between the building blocks. The initial weight loss (below $100^{\circ} \mathrm{C}$ ) is presumably due to the evaporation of the guest molecules such as acetone and water ${ }^{32}$.

The morphology of the synthesized samples was examined by scanning electron microscopy (SEM). Images of NRPOP-1 and NRPOP-2 (Fig. 2a,b) reveal that both polymers are composed of agglomerated spheres having different sizes. As the case of many POPs ${ }^{24,25,33}$, the two NRPOPs' PXRD patterns confirmed their amorphous nature by showing characteristic broad peaks (Fig. 2c).

Nitrogen adsorption-desorption experiments at $77 \mathrm{~K}$ were used to evaluate the porosity of the two POPs. As shown in Fig. $3 \mathrm{a}$, the isotherms increase sharply at the lower pressure region (below $0.01 \mathrm{P} / \mathrm{P}_{0}$ ) which is indicative of the microporosity nature of the NRPOPs ${ }^{34}$. The reversible adsorption-desorption isotherms with minimal hysteresis suggest that the two NRPOPs are rigid presumably due to the highly cross-linked structures (Table 1 summarizes the textural properties of both polymers ${ }^{28,31}$. The Brunauer-Emmet-Teller (BET) surface areas of NRPOP-1 and NRPOP-2 were determined to be $519 \mathrm{~m}^{2} \mathrm{~g}^{-1}$ and $456 \mathrm{~m}^{2} \mathrm{~g}^{-1}$, respectively. The lower surface area and the smaller total pore volume $\left(0.675 \mathrm{~cm}^{3} \mathrm{~g}^{-1}\right)$ of the NRPOP-2 polymer are presumably due to the existence of the phenyl group in the 1,4-bis-(2,4-diamino-1,3,5-triazine)benzene building block, leading to conformational flexibility in the polymer ${ }^{3,33}$. The pore size distribution (PSD) of the polymers were determined 


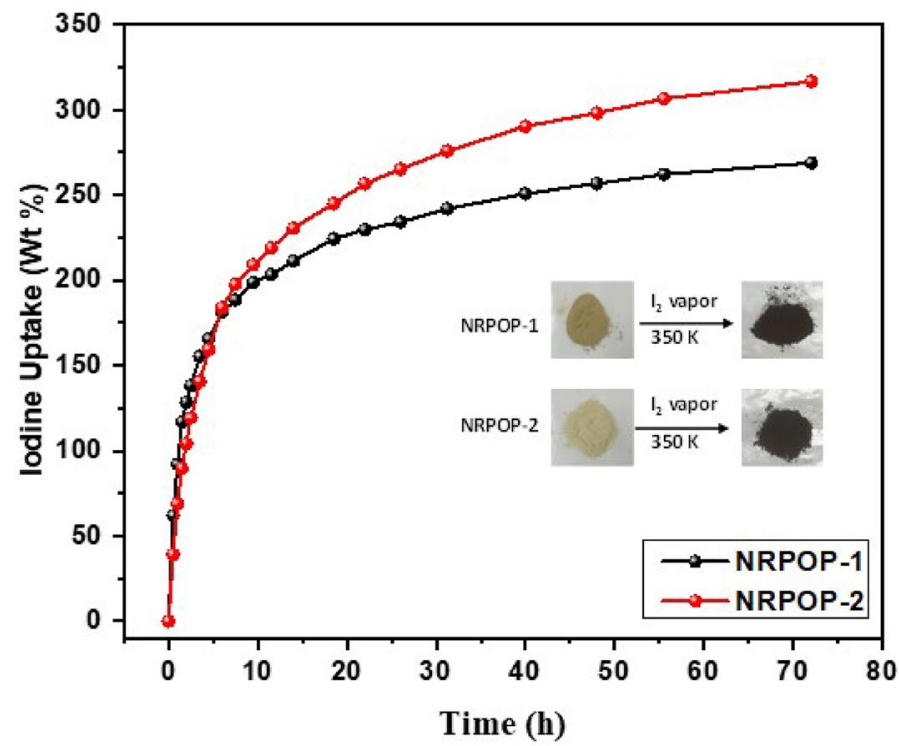

Figure 4. Gravimetric iodine uptake by NRPOPs overtime at $80{ }^{\circ} \mathrm{C}$ and ambient pressure; inset: photographs show the color change of NRPOPs after the exposure with iodine vapor.

using the nonlocal-density-functional theory (NLDFT). The highest peaks were observed at 1.2 (NRPOP-1) and $1.3 \mathrm{~nm}$ (NRPOP-2) as presented in Fig. $3 \mathrm{~b}$.

lodine capture and adsorption of the NRPOPs. The iodine capturing capacity of the NRPOPs was tested by adding known amounts of the NRPOPs into glass vials and placing them into a sealed glass jar containing an excess amount of iodine crystals. The system was heated up to $350 \mathrm{~K}$ and 1 bar and the samples weights were measured after some time intervals. In the first $8 \mathrm{~h}$, both materials exhibited a significant increase in iodine adsorption ( $200 \mathrm{wt} \%)$ presumably due to the vast abundance of adsorbing sites with an apparent color change from yellow to black (Fig. 4 inset). Afterward, the loading capacity continued to increase but at slower rates and reached equilibrium after $72 \mathrm{~h}$ of contact time with maximum iodine uptakes of 269 and $317 \mathrm{wt} \%$ for NRPOP-1 and NRPOP-2, respectively (Fig. 4). Such capacities are either higher or comparable to other porous aminallinked porous polymers (Table S1). The obtained high iodine capacities of the two NRPOPs can be attributed to the presence of the electron deficient triazine moieties and - $\mathrm{NH}$ - unites which enhance the dipole-dipole interaction between the polymers structures and the iodine. In addition, the synergetic functionalities of the NRPOPs having both $\pi$-conjugated surfaces and rich-heteroatoms ( $\mathrm{N}$ and $\mathrm{S}$ ) provide another important favourable interaction between the adsorbates and the adsorbents ${ }^{35,36}$. The higher iodine uptake by NRPOP-2 can be attributed to the extra phenyl ring in its structure. Previous studies have shown that porous polymers bearing a plentiful number of phenyl rings enhance their affinity to adsorbates because they provide an electron-rich environment that enables stronger iodine adsorbent interactions $3,25,27,37$.

The iodine loading capacities were also studied by the TGA of the iodine loaded ( $\left.\mathrm{I}_{2} @ N R P O P s\right)$. The $\mathrm{I}_{2}$-NRPOPs curves show steep weight loss in the temperature range of 90 to $350{ }^{\circ} \mathrm{C}$ (Fig. S2). Such weight loss is presumably due to the release of the encapsulated iodine since the pristine polymers are stable under this temperature range. The weight loss of the iodine was estimated to be 2.65 and $2.71 \mathrm{~g} \mathrm{~g}^{-1}$ for $\mathrm{I}_{2} @ \mathrm{NRPOP}-1$ and $\mathrm{I}_{2} @$ NRPOP-2, respectively, which are close to the equilibrium adsorption values especially for that of NRPOP1 . The difference in the weight loss and the saturated value of the NRPOP-2 is probably due to the incomplete release of the captured iodine due to its stronger interaction with the polymer as reasoned above. Major peaks in the IR spectra of the $\mathrm{I}_{2} @ N R P O P$ s revealed a significant shift compared to NRPOPs (Fig. S3). For example, the secondary amine bands of the $\mathrm{I}_{2} @ N R P O P-1$ and $\mathrm{I}_{2} @ N R P O P-2$ are red shifted by 84 and $60 \mathrm{~cm}^{-1}$, while the characteristic peaks of the triazine blue shifted by 49 and $66 \mathrm{~cm}^{-1}$, respectively. Such observations clearly suggest that the iodine is being captured at the secondary amine, aromatic rings, and triazine units of the NRPOPs. Similar to these shifts in the IR spectra have been observed in the iodine loaded HCMP-3 and iodine-doped PANi, and were attributed to the formation of charge transfer (CT) complexes between the polymers and polyiodide ions $^{38,39}$. PXRD measurements of the $\mathrm{I}_{2} @ N R P O P s$ showed only broad peaks indicating their amorphous nature and absence of pristine iodine crystals (Fig. S4) and hence suggesting the iodine is encapsulated inside the pores.

In addition to the gravimetric iodine vapor uptake, the ability of the synthesized polymers to adsorb iodine from the solution was investigated. In a typical experiment, $3 \mathrm{mg}$ of the NRPOPs were immersed into $300 \mathrm{mg} \mathrm{L}^{-1}$ cyclohexane solutions of iodine $(3 \mathrm{~mL})$. With time, the color of the solutions gradually faded away from purple to light pink/colorless, indicating the removal of iodine from the solutions (Fig. 5a, inset). UV-Vis spectroscopic measurements were carried out to quantify the amount of the removed iodine and to monitor the progress of the adsorption rates. Figure $5 \mathrm{a}$ displays a plot of iodine percentage removal vs. time that clearly shows that the adsorption kinetics consists of two stages: (1) a rapid initial increase in the adsorptions in the first $2 \mathrm{~h}$ and (2) 

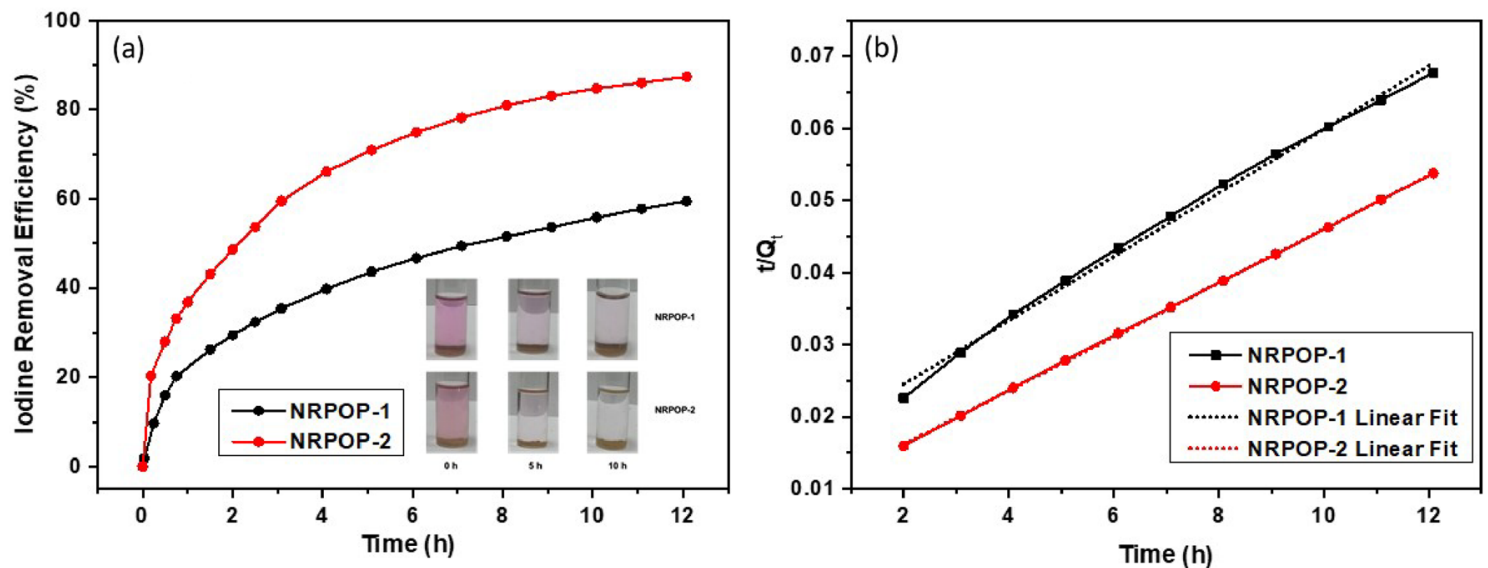

Figure 5. Iodine percentage removal from $300 \mathrm{mg} \mathrm{L}^{-1}$ iodine solution of cyclohexane by NRPOPs; Inset: photographs showing solutions color change after immersing NRPOPs into the solutions (a); pseudo-secondorder kinetic fitting for iodine adsorption experimental data $(\mathbf{b})$.
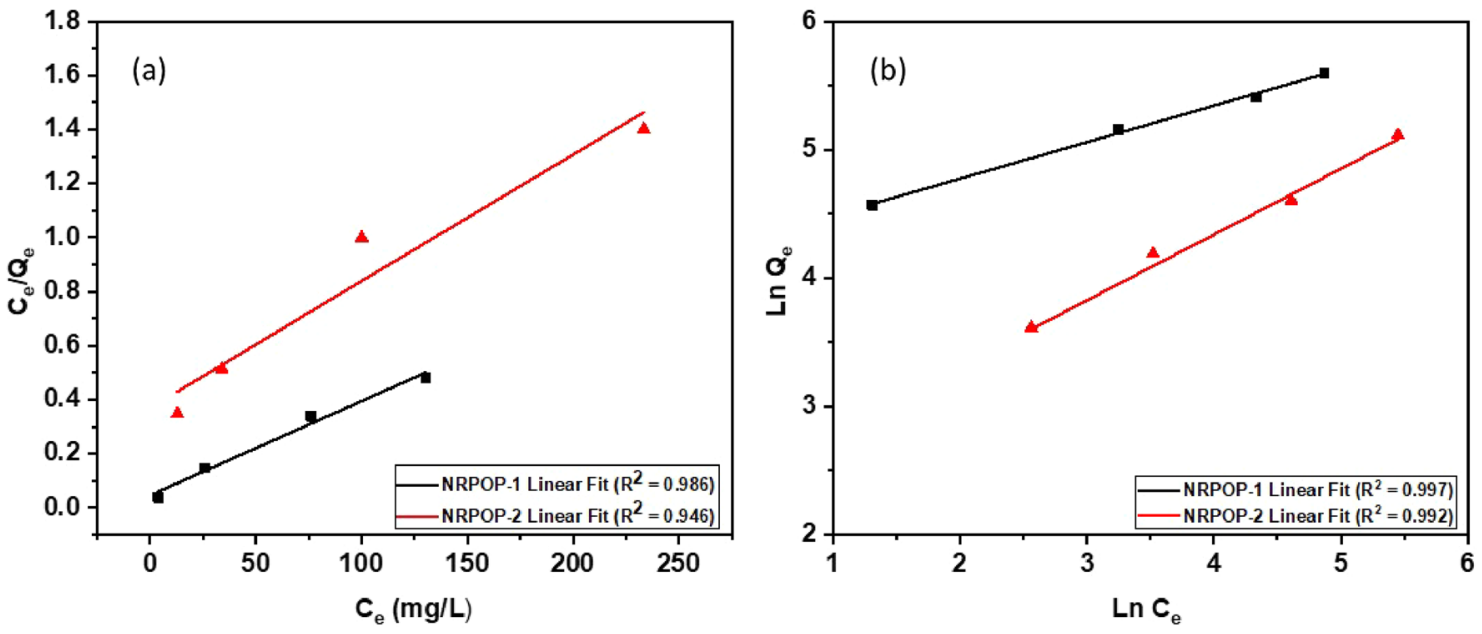

Figure 6. Iodine adsorption by NRPOPs isotherms fitted to Langmuir model (a) and Freundlich model (b). $R^{2}$ is the correlation coefficient of the linear fit.

a slow steady increase afterward until equilibrium was reached. NRPOP-1 and NRPOP-2 exhibited an iodine removal efficiency of $59 \%$ (177 $\mathrm{mg} \mathrm{g}^{-1}$ uptake) and $87 \%\left(261 \mathrm{mg} \mathrm{g}^{-1}\right.$ uptake) from $300 \mathrm{mg} \mathrm{L}^{-1}$ cyclohexane solutions, respectively. The lower iodine uptake compared to that in the vapor phase is presumably due to the encapsulation of solvent molecules inside the NRPOPs pores. The faster and higher iodine removal efficiency of the NRPOP-2 can be related to the richer conjugated structure because of the presence of phenyl rings in its structure, and hence, leading to an enhanced iodine-host polymer interactions ${ }^{3,40}$. Recently, theoretical calculation (DFT) for iodine uptake studies have been reported that substantiate the experimental results ${ }^{41}$.

The obtained kinetics results were fitted into pseudo-first and pseudo-second-order kinetic equations (SI). The calculated $R^{2}$ values (Table $\mathrm{S} 2$ ) indicate that the iodine adsorption kinetics fit better a pseudo-second-order kinetic model for both NRPOPs (Figs. 5b, S5). Such results suggest that the adsorption of iodine is governed by the chemisorption process ${ }^{39,40}$. This is attributed to the presence of high interaction between the iodine molecules and the surface of the NRPOPs. The presence of aromatic rings and electron-rich heteroatoms in the NRPOPs provide electron-donating moieties that are proficient in donating electrons to the electron-accepting iodine molecules, leading to charge transfer (CT) interactions and thus promoting higher iodine capturing capacity ${ }^{3,24}$. Previous reports indicated that such CT interactions are enhanced by the existence of - $\mathrm{NH}$ - units in the host structure ${ }^{25,40}$. This could be an additional factor for the excellent iodine uptake given the abundant $-\mathrm{NH}-\mathrm{con}$ taining pores of the NRPOPs.

To gain more insight into the adsorption mechanism of the iodine by the NRPOPs, we carried out adsorption equilibrium experiments by adding $1 \mathrm{mg}$ of each NRPOPs into a range of $1 \mathrm{~mL}$ iodine solutions of various concentrations at $298 \mathrm{~K}$. After $72 \mathrm{~h}$ of soaking, the residual concentrations of iodine in the solutions were determined by UV- Vis spectroscopy. The obtained isotherm data were then fitted into the two widely utilized isotherm models: Langmuir and Freundlich (Fig. 6a,b). The Langmuir model suggests a monolayer adsorption on the surface with homogenous distribution in the active sites, whereas the Freundlich model suggests a reversible 


\begin{tabular}{|l|l|l|l|l|l|l|}
\hline & \multicolumn{4}{|l}{ Langmuir } & \multicolumn{3}{l|}{ Freundlich } \\
\cline { 2 - 7 } & $\mathbf{Q}_{\mathbf{m}}$ & $\mathbf{K}_{\mathbf{l}}$ & $\mathbf{R}^{\mathbf{2}}$ & $\mathbf{N}$ & $\mathbf{K}_{\mathbf{f}}$ & $\mathbf{R}^{\mathbf{2}}$ \\
\hline NRPOP-1 & 285.7 & 0.749 & 0.986 & 3.52 & 67.10 & 0.997 \\
\hline NRPOP-2 & 212.8 & 0.0127 & 0.946 & 1.99 & 10.54 & 0.992 \\
\hline
\end{tabular}

Table 2. Langmuir and Freundlich parameters for the adsorption of Iodine. Langmiur model: $\mathrm{Q}_{\mathrm{m}}=$ maximum monolayer coverage capacity $\left(\mathrm{mg} \mathrm{g}^{-1}\right), \mathrm{K}_{\mathrm{l}}=$ Langmuir isotherm constant $\left(\mathrm{L} \mathrm{mg}^{-1}\right)$; Freundlich model: $\mathrm{K}_{\mathrm{f}}=$ Freundlich isotherm constant $\left(\mathrm{mg} \mathrm{g}^{-1}\right), \mathrm{N}=$ adsorption intensity; $R^{2}$ is the correlation coefficient of the linear fit.
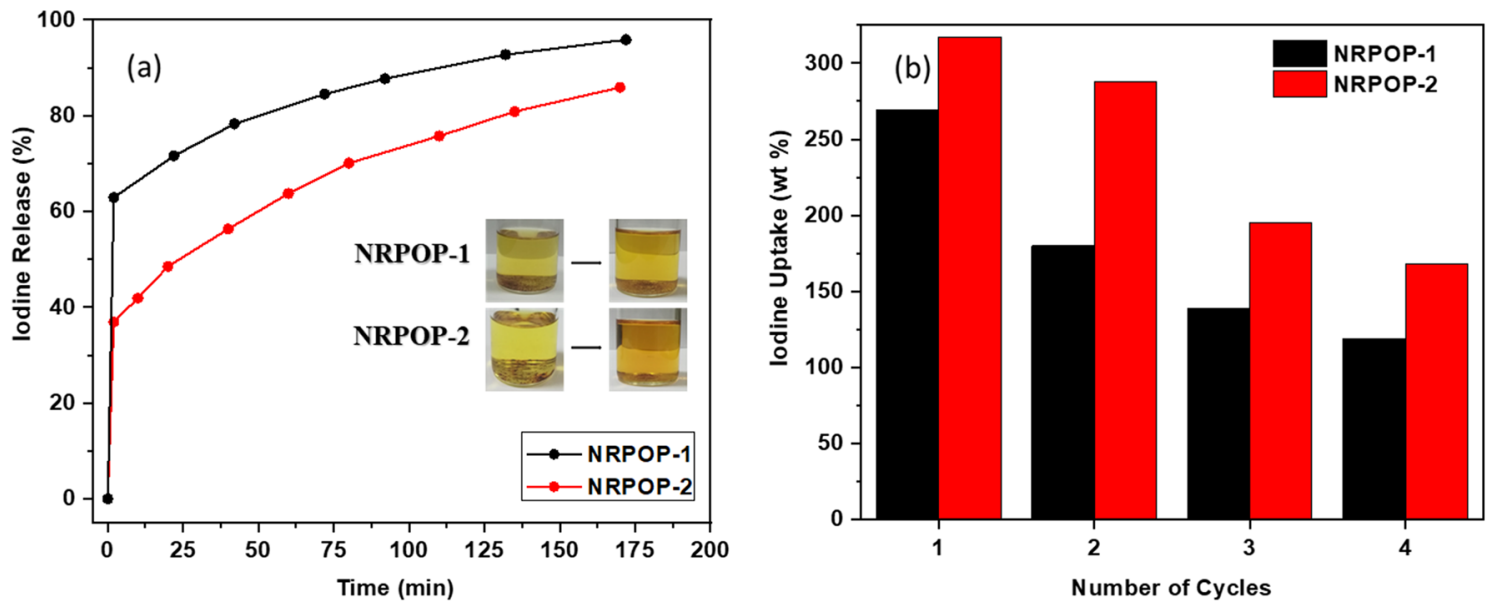

Figure 7. Percentage of iodine released from the NRPOPs by immersion in ethanol (a) and recyclability of NRPOPs for iodine vapor uptake (b).

heterogeneous multilayer interaction ${ }^{24,25}$. The extracted isotherm parameters and linear regression coefficients are displayed in Table 2. The higher $R^{2}$ values for the Freundlich model indicate that the equilibrium adsorption isotherms can be well fitted using this model. Accordingly, the adsorption of the iodine by NRPOPs can be described as a heterogeneous multilayer reversible adsorption process. Such conclusion is supported by the presence of different iodine adsorption sites within the NRPOPs that include triazine, dithiophene, and -NH- units ${ }^{3}$.

lodine release from iodine-loaded NRPOPs and potential applications. The encapsulated iodine can be readily released upon placing the $\mathrm{I}_{2} @ \mathrm{NRPOPs}$ in polar organic solvents such as ethanol at room temperature. To evaluate the releasing process, $5 \mathrm{mg}$ of the $\mathrm{I}_{2} @ \mathrm{NRPOPs}$ were immersed in ethanol $(50 \mathrm{~mL})$ as time progressed, the color of the solution gradually changed from colourless to brown indicating the release of the encapsulated iodine (Fig. 7a inset). The amount of iodine released into the ethanol solution was monitored by UV-vis measurements (Fig. 7a) at different time intervals. $\mathrm{I}_{2} @ N R P O P-1$ and $\mathrm{I}_{2} @ N R P O P-2$ exhibited a linear and quick release of iodine in the first 2 min followed by slow but steady increase over almost $3 \mathrm{~h}$ to reach a maximum iodine release efficiency of 95.8 and $85.9 \%$, respectively. The linear initial increase implies pseudozero-order kinetics which is inductive of host-guest interactions ${ }^{37}$. It is interesting to note that the slower and lower iodine release efficiency of $\mathrm{I}_{2} @ N R P O P-2$ is consistent with the higher iodine capture because of the strong $\pi$-conjugation interactions which facilitate the iodine capture, but also reduce the release efficiency and prolong the release time.

Considering the efficient release of iodine by the loaded polymers, this feature of $\mathrm{I}_{2} @ N R P O P s$ was further investigated for two potential applications. For the first application, the release of iodine from the polymers allows for another cycle of iodine loading which enables the compounds to act as transport carriers to move iodine from one medium to another. For example, NRPOPs can be used to remove pollutant radioactive iodine from the environment and release it into controlled containers with subsequent reuse of the polymers for more cycles. For the second application, the iodine-loaded polymers can be used to deliver iodine into a specific environment with controlled quantities. For example, since iodine is known to have antiseptic properties effective against a broad range of both gram-positive and negative bacteria ${ }^{42,43}, \mathrm{I}_{2} @ N R P O P$ can be used as a delivery vehicle of iodine in such areas as wound dressing or bandage to prevent bacterial infections.

Accordingly, we investigated the reusability of the polymers by first soaking samples of $\mathrm{I}_{2} @ N R P O P s$ in ethanol to release the iodine and then applied thermal activation to release solvent molecules from the pores of the polymers. The regenerated samples were then subjected to gravimetric iodine uptake experiments (as described above). The NRPOP-1 and NRPOP-2 were able to capture up to 119 and $168 \mathrm{~g} \mathrm{~g}^{-1}$ of iodine, respectively, which correspond to 44 and $53 \%$ retention of their initial iodine uptake capacity (Fig. $7 \mathrm{~b}$ ) after four loadingrelease-regeneration cycles. The decrease in the loading efficiency is probably due to the strong iodine-polymers 


\begin{tabular}{|l|l|l|l|l|}
\hline & Control & EC $^{\mathbf{a}}$ & PSA $^{\mathbf{b}}$ & ML $^{\mathbf{c}}$ \\
\hline NRPOP-1 & 0 & $0.9 \pm 0.1$ & $1.8 \pm 0.1$ & $2.7 \pm 0.2$ \\
\hline NRPOP-2 & 0 & $0.9 \pm 0.1$ & $1.8 \pm 0.1$ & $2.0 \pm 0.1$ \\
\hline
\end{tabular}

Table 3. Diameters $(\mathrm{cm})$ of inhibition rings of the bacteria on a petri dish by the loaded polymers.

${ }^{\mathrm{a}}$ Escherichia coli (MicroKwik Culture \# 155065A). ${ }^{\mathrm{b}}$ Pseudomonas aeruginosa (MicroKwik Culture \# 155250A).

${ }^{\mathrm{c}}$ Micrococcus luteus (MicroKwik Culture \# 155155A).

interactions, causing the incomplete release of the encapsulated time. The recycled NRPOPs were characterized further with PXRD and FTIR spectroscopy (Figs. S6 and S7). The results show the excellent stability and robustness of the POPs. The small shift toward the right side in the PXRD peaks after the fourth cycle is probably due to the incomplete release of the captured iodine by the stronger interaction between the polymer and iodine molecules.

Antibacterial activity of the captured iodine. In order to investigate the potential application of $\mathrm{I}_{2} @$ NRPOPs as a delivery vehicle of iodine for antiseptic action, we tested the ability of iodine-loaded NRPOPs to inhibit the growth of three types of bacteria, Escherichia coli (EC), Pseudomonas aeruginosa (PSA), and Micrococcus luteus $(\mathrm{ML})$ using agar diffusion method. A sample $(1 \mathrm{mg})$ of each iodine-loaded polymer was placed in the center of an agar petri dish seeded with the specific bacterial stain and the sample was covered by a wet paper disk. After incubation for $24 \mathrm{~h}$, the diameter of the growth-inhibited zone was measured and compared to the control samples (polymers without iodine). The results presented in Table 3 (Fig. S8) reveal that both iodine-loaded NRPOP-1 and NRPOP-2 inhibited the growth of gram-negative bacteria, PSA and EC, and grampositive bacteria ML with similar extents while the polymer alone (control) had no effect on bacterial growth. These results suggest that a significant amount of loaded iodine that was released from the polymer during the incubation time $(24 \mathrm{~h})$ prevented the growth of bacteria ${ }^{44}$, hence supporting the idea of potential application of NRPOPs as delivery vehicles of iodine for antiseptic applications.

\section{Conclusion}

In summary, we synthesized two nitrogen rich aminal-linked porous organic polymers, NRPOP-1 and NRPOP-2, by metal free-Schiff base polycondensation reaction of 1,3,5-triazine-2,4,6-triamine (melamine) and 1,4-bis-(2,4diamino-1,3,5-triazine)-benzene with thieno[2,3-b]thiophene-2,5-dicarboxaldehyde to attain the synergism of iodine uptake and the successive antibacterial use of the adsorbed iodine. Both polymers displayed excellent iodine vapor capturing capacity and were able to remove over $87 \%$ of dissolved iodine from $300 \mathrm{mg} \mathrm{L}^{-1}$ solution. The presence of phenyl units in the NRPOP-2 structure proved to be a major factor for the higher iodine uptake. The iodine-loaded polymers exhibited good antibacterial activity against the Micrococcus luteus (ML), Escherichia coli (EC), and Pseudomonas aeruginosa (PSA). The excellent iodine uptake and the effectiveness as antibacterial agents, NRPOPs make them promising candidates for environmental remediation and drug-delivery applications.

Received: 14 November 2021; Accepted: 2 February 2022

Published online: 16 February 2022

\section{References}

1. Cicia, G., Cembalo, L., Del Giudice, T. \& Palladino, A. Fossil energy versus nuclear, wind, solar and agricultural biomass: Insights from an Italian national survey. Energy Policy 42, 59-66 (2012).

2. Rabilloud, X. Comments on 'prevented mortality and greenhouse gas emissions from historical and projected nuclear power'. Environ. Sci. Technol. 47, 13896-13899 (2013).

3. Abdelmoaty, Y. H., Tessema, T. D., Choudhury, F. A., El-Kadri, O. M. \& El-Kaderi, H. M. Nitrogen-rich porous polymers for carbon dioxide and iodine sequestration for environmental remediation. ACS Appl. Mater. Interfaces 10, 16049-16058 (2018).

4. Xie, W., Cui, D., Zhang, S. R., Xu, Y. H. \& Jiang, D. L. Iodine capture in porous organic polymers and metal-organic frameworks materials. Mater. Horizons 6, 1571-1595 (2019).

5. Alsbaiee, A. et al. Rapid removal of organic micropollutants from water by a porous $\beta$-cyclodextrin polymer. Nature 529, 190-194 (2016).

6. Chapman, K. W., Chupas, P. J. \& Nenoff, T. M. Radioactive iodine capture in silver-containing mordenites through nanoscale silver iodide formation. J. Am. Chem. Soc. 132, 8897-8899 (2010).

7. Subrahmanyam, K. S. et al. Chalcogenide aerogels as sorbents for radioactive iodine. Chem. Mater. 27, 2619-2626 (2015).

8. Hetzel, B. S. \& Dunn, J. T. The iodine deficiency disorders: Their nature and prevention. Annu. Rev. Nutr. 9, 21-38 (1989).

9. Chen, Y. et al. Preparation, property of the complex of carboxymethyl chitosan grafted copolymer with iodine and application of it in cervical antibacterial biomembrane. Mater. Sci. Eng. C 67, 247-258 (2016).

10. Bürgi, H. Iodine excess. Best Pract. Res. Clin. Endocrinol. Metab. 24, 107-115 (2010).

11. Atwater, J. E., Sauer, R. L. \& Schultz, J. R. Numerical simulation of iodine speciation in relation to water disinfection aboard manned spacecraft I. Equilibria. J. Environ. Sci. Health Part A Toxic Hazard. Subst. Environ. Eng. 31, 1965-1979 (1996).

12. Lamme, E. N., Gustafsson, T. O. \& Middelkoop, E. Cadexomer-iodine ointment shows stimulation of epidermal regeneration in experimental full-thickness wounds. Arch. Dermatol. Res. 290, 18-24 (1998).

13. Goodwin, M. J. et al. Halogen and hydrogen bonding in povidone-iodine and related co-phases. Cryst. Growth Des. 17, 5552-5558 (2017).

14. Sriwilaijaroen, N. et al. Mechanisms of the action of povidone-iodine against human and avian influenza A viruses: Its effects on hemagglutination and sialidase activities. Virol. J. 6, 1-10 (2009). 
15. Papadopoulou, E. L. et al. Antibacterial bioelastomers with sustained povidone-iodine release. Chem. Eng. J. 347, 19-26 (2018).

16. Gao, B., Wang, Z., Liu, Q. \& Du, R. Immobilization of povidone-iodine on surfaces of silica gel particles and bactericidal property. Colloids Surf. B Biointerfaces 79, 446-451 (2010).

17. Das, S., Heasman, P., Ben, T. \& Qiu, S. Porous organic materials: Strategic design and structure-function correlation. Chem. Rev. 117, 1515-1563 (2017).

18. Rabbani, M. G., Sekizkardes, A. K., El-Kadri, O. M., Kaafarani, B. R. \& El-Kaderi, H. M. Pyrene-directed growth of nanoporous benzimidazole-linked nanofibers and their application to selective $\mathrm{CO}_{2}$ capture and separation. J. Mater. Chem. 22, 25409-25417 (2012).

19. Qian, X. et al. Novel N-rich porous organic polymers with extremely high uptake for capture and reversible storage of volatile iodine. J. Hazard. Mater. 338, 224-232 (2017).

20. Shen, Y., Ni, W. X. \& Li, B. Porous organic polymer synthesized by green diazo-coupling reaction for adsorptive removal of methylene blue. ACS Omega 6, 3202-3208 (2021).

21. Huang, L. et al. Nanoarchitectured porous organic polymers and their environmental applications for removal of toxic metal ions. Chem. Eng. J. 408, 127991 (2021).

22. Li, G., Yao, C., Wang, J. \& Xu, Y. Synthesis of tunable porosity of fluorine-enriched porous organic polymer materials with excellent $\mathrm{CO}_{2}, \mathrm{CH}_{4}$ and iodine adsorption. Sci. Rep. 7, 1-8 (2017).

23. Xu, Y., Jin, S., Xu, H., Nagai, A. \& Jiang, D. Conjugated microporous polymers: Design, synthesis and application. Chem. Soc. Rev. 42, 8012-8031 (2013).

24. Sabri, M. A., Al-Sayah, M. H., Sen, S., Ibrahim, T. H. \& El-Kadri, O. M. Fluorescent aminal linked porous organic polymer for reversible iodine capture and sensing. Sci. Rep. 10, 1-11 (2020).

25. Sen, S., Al-Sayah, M. H., Mohammed, M. S., Abu-Abdoun, I. I. \& El-Kadri, O. M. Multifunctional nitrogen-rich aminal-linked luminescent porous organic polymers for iodine enrichment and selective detection of $\mathrm{Fe}^{3+}$ ions. J. Mater. Sci. 55, 10896-10909 (2020).

26. Ren, F. et al. Novel thiophene-bearing conjugated microporous polymer honeycomb-like porous spheres with ultrahigh iodine uptake. Chem. Commun. 52, 9797-9800 (2016).

27. Jiang, Q., Huang, H., Tang, Y., Zhang, Y. \& Zhong, C. Highly porous covalent triazine frameworks for reversible iodine capture and efficient removal of dye. Ind. Eng. Chem. Res. 57, 15114-21512 (2018).

28. Song, W. C., Xu, X. K., Chen, Q., Zhuang, Z. Z. \& Bu, X. H. Nitrogen-rich diaminotriazine-based porous organic polymers for small gas storage and selective uptake. Polym. Chem. 4, 4690-4696 (2013).

29. Ren, Y. Y. et al. Nitrogen-rich porous polyaminal network as a platform for iodine adsorption through physical and chemical interaction. J. Appl. Polym. Sci. 135, 6-11 (2018).

30. Schwab, M. G. et al. Catalyst-free preparation of melamine-based microporous polymer networks through Schiff base chemistry. J. Am. Chem. Soc. 131, 7216-7217 (2009).

31. Weng, J. Y., Xu, Y. L., Song, W. C. \& Zhang, Y. H. Tuning the adsorption and fluorescence properties of aminal-linked porous organic polymers through N-heterocyclic group decoration. J. Polym. Sci. Part A Polym. Chem. 54, 1724-1730 (2016).

32. Mohan, A. et al. Silver nanoparticles impregnated $\mathrm{pH}$-responsive nanohybrid system for the catalytic reduction of dyes. Microporous Mesoporous Mater. 303, 110260 (2020).

33. Sabri, M. A. et al. Simultaneous adsorption and reduction of $\mathrm{cr}(\mathrm{Vi})$ to $\mathrm{cr}(\mathrm{iii})$ in aqueous solution using nitrogen-rich aminal linked porous organic polymers. Sustainability 13, 1-13 (2021).

34. Ben, T. et al. Gas storage in porous aromatic frameworks (PAFs). Energy Environ. Sci. 4, 3991-3999 (2011).

35. Bhunia, A. et al. A photoluminescent covalent triazine framework: $\mathrm{CO}_{2}$ adsorption, light-driven hydrogen evolution and sensing of nitroaromatics. J. Mater. Chem. A 4, 13450-13457 (2016).

36. Guo, Z. et al. Amorphous porous organic polymers based on Schiff-base chemistry for highly efficient iodine capture. Chem. Asian J. 13, 2046-2053 (2018).

37. Chen, Y. et al. Synthesis of conjugated microporous polymer nanotubes with large surface areas as absorbents for iodine and $\mathrm{CO}_{2}$ uptake. J. Mater. Chem. A 3, 87-91 (2015).

38. Liao, Y., Weber, J., Mills, B. M., Ren, Z. \& Faul, C. F. J. Highly efficient and reversible iodine capture in hexaphenylbenzene-based conjugated microporous polymers. Macromolecules 49, 6322-6333 (2016).

39. Mathai, C. J., Saravanan, S., Anantharaman, M. R., Venkitachalam, S. \& Jayalekshmi, S. Effect of iodine doping on the bandgap of plasma polymerized aniline thin films. J. Phys. D. Appl. Phys. 35, 2206-2210 (2002).

40. Yang, Y. et al. Insight into volatile iodine uptake properties of covalent organic frameworks with different conjugated structures. J. Solid State Chem. 279, 120979 (2019).

41. Pourebrahimi, S. \& Pirooz, M. Reversible iodine vapor capture using bipyridine-based covalent triazine framework: Experimental and computational investigations. Chem. Eng. J. Adv. 8, 100150 (2021).

42. Properties, P. I. \& Action, M. crossm in infection control and Staphylococcus aureus ecolonization. Oral Oncol. 105, 581-593 (2020).

43. Bigliardi, P. L. et al. Povidone iodine in wound healing: A review of current concepts and practices. Int. J. Surg. 44, 260-268 (2017).

44. Borjihan, Q. et al. Pyrrolidone-based polymers capable of reversible iodine capture for reuse in antibacterial applications. J. Hazard. Mater. 384, 121305 (2020).

\section{Acknowledgements}

Funding was provided by the American University of Sharjah, grant \# EFRG18-GER-CAS-67). The work in this paper was also supported, in part, by the Open Access Program from the American University of Sharjah. This paper represents the opinions of the author(s) and does not mean to represent the position or opinions of the American University of Sharjah.

\section{Author contributions}

O.M.E-K conceived and supervised the project, designed the experiments, and wrote the manuscript. M.H.A. performed the antibacterial study and analysed the data. A.M. and A.A. Setup and performed the iodine uptake experiments and carried out the kinetic and equilibrium studies. All authors contributed in preparing and finalizing the manuscript.

\section{Competing interests}

The authors declare no competing interests.

Additional information

Supplementary Information The online version contains supplementary material available at https://doi.org/ 10.1038/s41598-022-06671-0. 
Correspondence and requests for materials should be addressed to O.M.E.-K.

Reprints and permissions information is available at www.nature.com/reprints.

Publisher's note Springer Nature remains neutral with regard to jurisdictional claims in published maps and institutional affiliations.

(c) (i) Open Access This article is licensed under a Creative Commons Attribution 4.0 International cc) License, which permits use, sharing, adaptation, distribution and reproduction in any medium or format, as long as you give appropriate credit to the original author(s) and the source, provide a link to the Creative Commons licence, and indicate if changes were made. The images or other third party material in this article are included in the article's Creative Commons licence, unless indicated otherwise in a credit line to the material. If material is not included in the article's Creative Commons licence and your intended use is not permitted by statutory regulation or exceeds the permitted use, you will need to obtain permission directly from the copyright holder. To view a copy of this licence, visit http://creativecommons.org/licenses/by/4.0/.

(C) The Author(s) 2022 